\title{
Moderate Hypoxia Exhibits Increased Endothelial Progenitor Vessel-forming Ability However Gestational Diabetes Caused to Impede Compensatory Defense Reaction
}

\author{
U. Deniz Dincer ${ }^{1,2,3}$ \\ ${ }^{I}$ Department of Pharmacology, School of Pharmacy, ${ }^{2}$ Department of Clinical Pharmacy, School of Pharmacy, \\ ${ }^{3}$ Department of Pharmacology, School of Medicine, Girne American University, Girne, North Cyprus via Mersin 10, Turkey
}

Endothelium represents a defense barrier and responds and integrates neuro humoral stimulus which describes as a compensatory mechanism. Endothelium formed with endothelial cells (ECs) and their progenitors. Endothelial progenitor cells (EPCs) represent minor subpopulation of mononuclear cells in the blood. During acute hypoxia, larger amount of EPCs mobilize into the peripheral blood and they directly contribute revascularization process. One of the subtypes of EPC is termed endothelial colony forming cells (ECFCs) which they possess de novo vessel-forming ability. The present study aims to investigate the role of hypoxia in EPCs functional and vessel-forming ability. Furthermore, it was investigated whether fetal exposure to a diabetic intrauterine environment influence EPCs adaptation ability. Human umbilical cord blood (HUCB) derived ECFCs were selected in all experimental procedures obtained from normal and gestational diabetes mellitus $(\mathrm{GDM})$ subjects via in vitro cell culture methods. Early passage $(<5)$ HUCB ECFCs obtain from GDM $(n ; 5)$ and control $(n ; 5)$ subjects were cultured with plates pre-coated with collagen in vitro $72 \mathrm{~h}$ hypoxic as well as normoxic condition. Endothelial, angiogenic and hypoxia associated gene specific primers designed to perform Real-time PCR. Senescenes assay conducted onto HUCB ECFCs to investigate their functional clonogenic ability. To quantify their vessel forming ability matrigel assay was applied. These data demonstrates that moderate hypoxia results increased vessel-forming ability and VEGFA expression in HUCB ECFCs obtained from control subjects. However, GDM caused to impede compensatory defense reaction against hypoxia which observed in control subjects. Thus, it illuminates beneficial information related future therapeutic modalities.

Keywords: Human endothelial progenitor cells, Moderate hypoxia, Human cord blood

Accepted for publication October 21, 2015, Published online May 30, 2016 Correspondence to U. Deniz Dincer

Department of Pharmacology, School of Pharmacy, Department of Clinical Pharmacy School of Pharmacy, Department of Pharmacology, School of Medicine, Girne American University, University Drive, 99428 Karmi Campus, Karaoglanoglu, Girne, North Cyprus via Mersin 10, Turkey

Tel: +90-392-6502000, Fax: +90-392-6502062

E-mail: denizdincer@gau.edu.tr

(c) This is an open-access article distributed under the terms of the Creative Commons Attribution Non-Commercial License (http://creativecommons.org/ licenses/by-nc/4.0/), which permits unrestricted non-commercial use, distribution, and reproduction in any medium, provided the original work is properly cited.

\section{Introduction}

Endothelium represents a protective barrier. It also responds and integrates against neuro humoral stimulus and environment. It describes as a compensatory defense mechanism, adaptation of existing pathological conditions in the human body. Endothelium formed with endothelial cells (ECs) and their progenitors. Studies have demonstrated that endothelial progenitors actually release bigger amount in disease conditions and mobilize into the peripheral blood and help to normalize vascular function (1-3). Endothelial progenitor cells (EPCs) are playing a 
tremendous role in endothelial injury to repair vascular function. To understand their actual role they were isolated from bone marrow, peripheral blood and human cord blood. However much, their isolation and purification are require developed cell culture technics with specific media and growth factors, their clonal proliferative potential and intrinsic in vivo blood vessel forming ability were generated different point of view (4). After high clonal EPCs injection was demonstrated that high tissue regeneration capacity and new vessel formation (1).

The blood outgrowth endothelial cells represent the most clonal proliferative potential capacity cell in the body which called endothelial colony forming cells (ECFCs) $(1,5)$. They are actually seldom in the circulating blood however present too many in the human umbilical cord blood (HUCB) $(1,5)$. They display in vivo vessel forming ability $(1,5)$. In recent years, investigators have focused on to generate high clonal proliferative potential human pluripotent stem cells (hPSCs) because they demonstrate self-repopulating capacity and vessel forming ability (1). They also display direct transplantable capability without an intervening period of in vitro cell culture. It truly indicates that they have able to re-endothelialize injured vessels furthermore generate new vessel formation thus improve blood flow $(1,5)$. Those facts definitely introduced a new therapeutic approach for many diseases such as myocardial infarction, stroke, ischemic limb injury and ischemic retinopathy (1). Beyond that therapeutically usage of endothelial progenitors as a fashionable topic in the clinics, their number, migration and function were also correlated with disease risk factor scores (6-10). One of the major correlations was cardiovascular disease which is low number of colonies reflects higher cardiovascular disease susceptibility (10). The other major correlations were increased number of colonies demonstrate opposite correlation with hypercholesterolemia, hypertension and type 2 diabetes mellitus (DM) (7-10).

Gestational diabetes mellitus (GDM), however, is a long term health consequences for her adult offspring which inducing development of metabolic syndrome, hypertension, type II diabetes mellitus and premature cardiovascular disease $(11,12)$. Some complications could be related with affected endothelial progenitors in adults during intrauterine development via fetal exposure to hyperglycemia which born to gestational diabetes mothers.

Our previous investigation indicated that endothelial progenitors demonstrate decreased adaptation ability against chronic hypoxia and fetal diabetic environment (11). They truly exhibited diminished proliferative capacity and vessel forming ability against chronic hypoxic condition which represents loss of their adaptation ability (11). Furthermore, it was demonstrated that GDM displays decreased EPCs proliferation capacity and vessel forming ability against chronic hypoxia. Actually, GDM itself increased risk for the development of cardiovascular diseases to their adult offspring. Thus, EPC's diminished adaptation ability could prevent development of new vessel formation as well as endothelial repair as a regular compensatory defense mechanism in adults which born to GDM mothers. During hypoxia a larger amount of EPCs are released and mobilized into the peripheral blood to constitute vasodilatation and increase vessel formation. Generally chronic hypoxia displays apoptosis-mediated cell death instead of generation of new vessel formation and endothelial repair (11). Consequently, the duration of hypoxia is the key moderators to generate re-endothelialize injured vessels and re-establish blood flow via new vessel formation as well as vasodilatation or to generate apoptosis via exhibits cell death.

This project aimed to investigate the adaptation ability of EPCs against moderate hypoxic environment. It gives beneficial information whether moderate hypoxia displays increased EPCs proliferation capacity and vessel forming capability or negatively displays apoptosis. Overall it indicates the role of endothelial progenitor cells onto vascular function in ischemic conditions such as coronary artery disease, pulmonary hypertension, obstructive hypoxic lung disease and sleep apnea disorders. As is well known, improvement of cardiovascular disease is some part related with healthy endothelium with their healthy endothelial progenitors and development of new vessel formation to re-establish blood flow. This project also help to generate new treatment approach if high clonal proliferative potential human pluripotent stem cells (hPSCs) such as HUCB ECFCs really worthy to normalize vessel function if they have dynamically self-repopulating capacity and vessel forming ability during ischemic conditions. Thus, we hypothesized that in vitro moderate hypoxia alters HUCB ECFCs functional and vascularization capacity furthermore whether fetal exposure to a diabetic intrauterine environment would result in failure of ECFCs adaptation ability to hypoxic environment.

\section{Materials and Methods}

\section{Clinical Data, Cord Blood Collection, Human Blood Samples}

This manuscript was prepared according to the Declaration of Helsinki based on the primary ethical obligations of patients especially to collect human cord blood samples 
(11-13). Pregnant subjects from uncomplicated pregnancies were chosen during outpatient clinics and/or delivery (Previously approved projects from Institutional Review Board [IRB], Department of Pediatrics, Indiana University School of Medicine, Indianapolis, IN, USA).

\section{Maternal Data}

GDM patients demonstrated symptoms of diabetes during their pregnancy (hyperglycemia; high blood glucose [BG] concentration [BG $\geq 11.1 \mathrm{mM} ; 200 \mathrm{mg} / \mathrm{dl}]$ or fasting plasma glucose $\geq 7.0 \mathrm{mM}$ [126 $\mathrm{mg} / \mathrm{dl}]$, or 2-hour plasma glucose $11.1 \mathrm{mM}$ [200 mg/dl] during an oral glucose tolerance test)) (11). HgbAlCs were around $39 \mathrm{mmol} / \mathrm{mol}$ $(<6 \%)$ and $117 \mathrm{mg} / \mathrm{dL}$ were estimated average glucose (eAG) (11).

\section{Endothelial colony forming cells (ECFC) isolation}

Endothelial colony forming cells (ECFC) and mononuclear cells (MNCs) isolated as described previously (11-15). Around $50 \mathrm{ml}$ blood collected from cord blood IRB approved patient's freshly delivered placentas by a research nurse or trained research personnel. MNCs were washed 3 times with EBM-2 medium (Cambrex, Walkersville, MD) supplemented with $10 \%$ fetal bovine serum (FBS; Hyclone, Logan, UT), 2\% penicillin/streptomycin, $5 \mathrm{mM}$ dextrose and $0.25 \mu \mathrm{g} / \mathrm{mL}$ amphotericin (Invitrogen, Grand Island, NY) (complete EBM-2 medium) and seeded onto six well tissue culture plates pre-coated with collagen. After $24 \mathrm{~h}$, adherent cells were washed and medium was changed daily until first ECFC colonies appear between 5 and 11 days as previously described (11-15). The number of colonies counted for each cord blood sample. Colonies trypsinized and plated in a flask pre coated with collagen. Cells passaged upon reaching 80 90\% confluence. Early passage $(<5)$ ECFC's cell lines used for all studies. For that purpose, 5 control and 5 GDM HUCB ECFCs selected (11).

\section{Moderate Hypoxia (72 h)}

Early passage $(<5)$ HUCB ECFCs obtain from GDM $(\mathrm{n} ; 5)$ and control $(\mathrm{n} ; 5)$ subjects were cultured with plates pre-coated with collagen under hypoxic (5\% $\mathrm{CO}_{2} / 94 \%$ $\mathrm{N}_{2}$ and $1 \% \mathrm{O}_{2}$ in a triple gas incubator) $(11,16,17)$ as well as normoxic condition for $72 \mathrm{~h}$ using $\mathrm{EGM}_{2}$ media with $10 \%$ FBS $(11,18)$.

\section{Characterization of HUCB ECFCs using Real-Time PCR}

HUCB ECFCs were identified with previously described cell culture methods (11-15). Endothelial specific primers designed to perform Real-time PCR to identify their endothelial origin (11). For that purpose; endothelial specific markers (VE-cadherin [CDH5, vascular endothelial-cadherin, $\mathrm{CdH} 5, \mathrm{Ca}^{++}$-dependent cell adhesion molecule, CD144], endothelial nitric oxide synthase [eNOS] and endothelial cell adhesion molecule [PECAM; CD 31]) were used $(11,19,20)$. Vascular endothelial growth factor A (VEGFA) and insulin-like growth factor 1 (IGF-1) primers were also designed to identify their pro-angiogenic properties (11, 21-23). Hypoxia specific markers adrenomedullin (ADM), G protein coupled activity modifying protein 2 and 3 receptor (RAMP 2 and RAMP 3) were designed to identify their acute hypoxia associated expressions $(24,25)$. However RAMP 3 did not express in HUCB ECFCs.

Total ribonucleic acid (RNA) was extracted and high quality samples were used as templates for synthesis of first-strand cDNAs as described previously (11, 26, 27). The total RNA quantity and quality were determined using the Experion ${ }^{\mathrm{TM}}$ semi-automated electrophoresis system (Bio-Rad Laboratories Inc., Hercules, CA, USA). RNA samples with distinct $18 \mathrm{~S}$ and $28 \mathrm{~S}$ ribosomal RNA fragments in the electropherogram were used to assess the RNA quality which described previously (28).

Real-time PCR reactions were performed for PECAM 1 (CD31), VE-cadherin (CD144), eNOS, VEGFA, IGF-1 and $\beta$-actin in duplicated with a custom designed SYBR Green mix [2.4 $\mu \mathrm{l}$ of $25 \mathrm{mM} \mathrm{MgCl}_{2}, 5 \mu \mathrm{l}$ of 1:10,000 dilution SYBR Green I (Molecular Probes) and $5 \mu \mathrm{l}$ of $1 \mathrm{nM}$ Fluorescein Calibration Dye (1 mM/L in DMSO, Bio-Rad) in $50 \mu \mathrm{l}$ of total reaction using Taq DNA polymerase (Promega) as described previously (11, 26-28)]. Primers were designed using Genomatix and (http:/www.genomatix.de/cgi-in/dialign/dialign.pl) the National Center for Biotechnology Information database (http://www3.ncbi. nlm.nih.gov/entrez/). The primer sequences used for transcripts were: PECAM 1 (CD31) sense GAGATGAGGTATGGGCTGGA and antisense TGCTGCATCAAGAGTGGTTC (accession number; AH002931); VE-Cadherin (CDH5) sense CCTACCAGCCCAAAGTGTGT and antisense GAGATGACCACGGGTAGGAA (accession number; NM_ 001795); eNOS sense ACCCTCACCGCTACAACATC and antisense GCTCATTCTCCAGGTGCTTC (accession number; NM_000603). ADM sense CGTCGGAGTTTCGAAAGAAG and antisense CCCTGGAAGTTGTTCATGCT (accession number; :NM_001124); RAMP 2 sense ACCAGATCCACTTTGCCAAC ${ }^{-}$and antisense GGTGGGGAAGTGGAGTAACA (accession number; NM_005854); VEGFA primers (sense CCAACTTCTGGGCTGTTCTC and antisense CСССТСТССТСТTССТTCTC) were designed with GENOMATIX program based on previously published accession 
numbers (BC011177, NM_001025366, NM_003376, NM_001025367, NM_001025368, NM_001025369, NM_001025370, NM_001033756). IGF-1 primers (sense CATGTCCTCCTCGCATCTCT and antisense ATACCCTGTGGGCTTGTTGA) were designed with GENOMATIX program based on previously published accession numbers (NM_000618, NM_001111283, NM_001111284, NM_001111285). Beta-actin primers (sense GGACTTCGAGCAAGAGATGG and antisense AGCACTGTGTTGGCGTACAG) were designed for integral control of the experiment (accession number NM_001101)(11).

Amplification was carried out with iCycler iQ multicolor real-time PCR detection system (Bio-Rad) as follows: 30 -s denaturation $\left(95^{\circ} \mathrm{C}\right)$ followed by 45 -s annealing and 1-min extension $\left(72^{\circ} \mathrm{C}\right)$, repeated for a total of 33 cycles. $\beta$-actin was amplified in each set of PCR reactions and served as an internal reference during quantitation to correct for operator and/or experimental variations. The data were analyzed in duplicated using the modified $2^{-\Delta \Delta C T}$ equation $(11,29)$.

\section{Modified $2^{-\Delta \Delta \mathrm{CT}}$ Equation \\ $\triangle \triangle \mathrm{CT}$ GDM Nor $=\left(\mathrm{Ct}_{\text {-gdm nor }}-\mathrm{Ct}_{\text {gdm nor BA }}\right)$ GDM nor- ( $\left.\mathrm{Ct}_{\text {-cont nor }}-\mathrm{Ct}_{\text {-cont nor } \mathrm{BA}}\right)$ Cont nor \\ $\triangle \triangle \mathrm{CT}$ Cont Hypx $=\left(\mathrm{Ct}_{\text {-cont hypx }}-\mathrm{Ct}_{\text {contr hypx } \mathrm{BA}}\right)$ Control Hypxc- $-\left(\mathrm{Ct}_{\text {-cont nor }}-\mathrm{Ct}_{\text {-cont nor } \mathrm{BA}}\right)$ Cont nor \\ $\triangle \triangle \mathrm{CT}$ GDM Hypx=(Ct.gdm hypx $\left.-\mathrm{Ct}_{\text {-gdm hypx BA }}\right) \mathrm{GDM}$ Hypxc-(Ct-gdm nor $\left.-\mathrm{Ct}_{\text {-gdm nor } \mathrm{BA}}\right) \mathrm{GDM}$ nor}

(Ct.gdm nor; $\mathrm{Ct}, \mathrm{GDM}$ samples under normoxic condition using interested in gene primers, $\mathrm{Ct}_{\text {gdm nor } \mathrm{BA}} \mathrm{Ct}, \mathrm{GDM}$ samples under normoxic condition using Beta actin primers, $\mathrm{Ct}_{\text {-cont nor; }} \mathrm{Ct}$, control samples under normoxic condition using interested in gene primers, $\mathrm{Ct}_{\text {cont nor } \mathrm{BA}} \mathrm{Ct}$, control samples under normoxic condition using Beta actin primers, $\mathrm{Ct}_{\text {cont hypx }} \mathrm{Ct}$, control samples under hypoxic

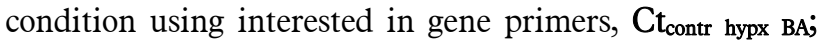
$\mathrm{Ct}$, control samples under hypoxic condition using Beta actin primers, $\mathrm{Ct}_{\text {gdm hypx; }} \mathrm{Ct}$, GDM samples under hypoxic

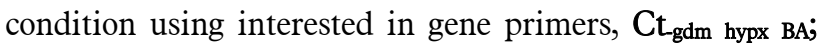
$\mathrm{Ct}$, GDM samples under hypoxic condition using Beta actin primers). The mean threshold cycle $\left(\mathrm{C}_{\mathrm{T}}\right)$ values for target and internal control ( $\beta$-actin) genes were determined in each sample. On the other hand, the formula was further modified to compare control and GDM samples under hypoxic and normoxic conditions. For that purpose specific gene primers as well as internal control Beta actin primers were run in each duplicated set (11).

\section{Senescence Assay}

Acidic $\beta$-galactosidase ( $\beta$-gal) assay conducted onto HUCB ECFCs to investigate their functional capacity against $72 \mathrm{~h}$ hypoxia $\left(5 \% \mathrm{CO}_{2} / 94 \% \mathrm{~N}_{2}\right.$ and $1 \% \mathrm{O}_{2}$ in a triple gas incubator) for each experimental group (C-HUCB ECFC-N; human umbilical cord blood ECFCs obtained from control pregnancies and plated under normoxic condition, C-HUCB ECFC-H; human umbilical cord blood ECFCs obtained from control pregnancies and plated under hypoxic condition, GDM-HUCB ECFC-N; human umbilical cord blood ECFCs obtained from GDM subjects and plated under normoxic condition, GDM-HUCB ECFC-H; human umbilical cord blood ECFCs obtained from GDM subjects and plated under hypoxic condition) which described previously (11). 2000 cells were seeded and triplicated into a well of 6 well plates for each experimental group and exposed to a $72 \mathrm{~h}$ hypoxic $(5 \%$ $\mathrm{CO}_{2} / 94 \% \mathrm{~N}_{2}$ and $1 \% \mathrm{O}_{2}$ in a triple gas incubator) as well as normoxic condition $(11,16-18) .72 \mathrm{~h}$ later hypoxic cells were fixed with $2 \%$ formaldehyde and $0.2 \%$ gluteraldehyde combination $(11,18)$. Then cells were stained with $\beta$-galactosidase solution ( $\mathrm{X}-\mathrm{Gal}+\beta$-galactosidase $1 \mathrm{mg} / \mathrm{ml}$ final concentration) and incubated normoxic cell culture for overnight. Next day, blue stained cells were counted under microscope. Increased stained with $\beta$-gal contributes the reduction of clonogenic capacity (11).

\section{Matrigel Assay}

After exposure to a $72 \mathrm{~h}$ hypoxia $\left(5 \% \mathrm{CO}_{2} / 94 \% \mathrm{~N}_{2}\right.$ and $1 \% \mathrm{O}_{2}$ in a triple gas incubator) ECFCs from each experimental group (C-HUCB ECFC-N, C-HUCB ECFC-H, GDM-HUCB ECFC-N, GDM-HUCB ECFC-H) were seeded and triplicated at 6000 cells/well in 96 well tissue culture plates coated with $35 \mu 1$ of matrigel (BD Biosciences, San Jose, CA, USA) $(11,12,18)$. After $6 \sim 10$ h later vessel forming capacity (number of closed network unit) quantitated with visual microscopy (Zeiss Axiovert 2 inverted microscope with a $5 \mathrm{X}$ CP-ACHROMAT/0.12 NA objective). Images collected for each experimental group with SPOT RT camera which previously described (11).

\section{Data and statistical analysis}

Differences among group values were evaluated by one-way analysis of variance followed by a Newman-Keuls test. Data are presented as means \pm SE. Results were considered significantly different at $\mathrm{p}<0.05$. Real-time PCR data was analyzed with the modified $2^{-\triangle \Delta C T}$ equation (11, 26-29). 


\section{Results}

\section{Characterization of HUCB ECFCs using Cell Culture and Real-Time PCR}

Flow cytometry demonstrated that the endothelial origin of human hematopoietic progenitor cells. They proved to be phenotypically indistinguishable from cultured ECs and possessed de novo vessel-forming ability. Cells were obtained from IRB-approved (under Declaration of Helsinki) ready HUCB ECFCs from the EPC Bank (The Herman B Wells Pediatric Research Center, Department of Pediatrics, Riley Pediatric Foundation and University

A

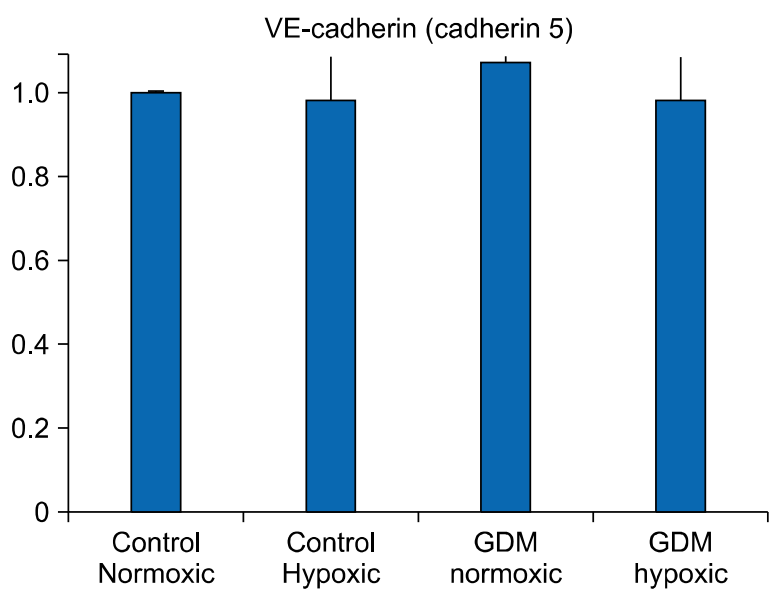

C

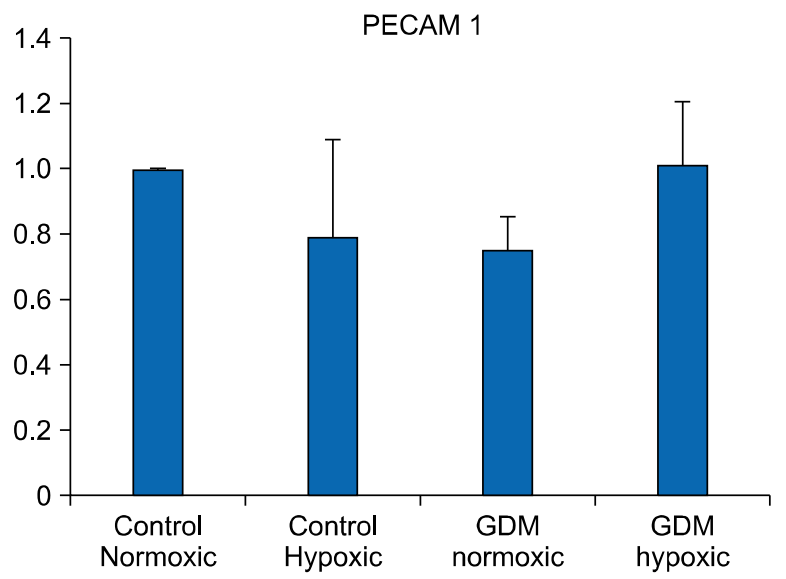

Pediatric Association, Indiana University School of Medicine, Indianapolis, IN, USA) which previously isolated and declared as ECFC (early passage $<5$ ). Umbilical cord blood MNCs plated onto collagen coated plate in endothelial specific media. Non-adherent cells discarded and cells appeared cobblestone formation.

Real-time PCR revealed that each HUCB ECFC expressed VE-cadherin (CD 144), VEGFA, PECAM 1 (CD 31), eNOS, IGF-1, ADM and RAMP 2 mRNAs under normoxic as well as hypoxic conditions. PECAM 1 (CD31) only expresses in endothelial cells and, in the same way, VE-cadherin is exclusively specific marker. All samples in

B

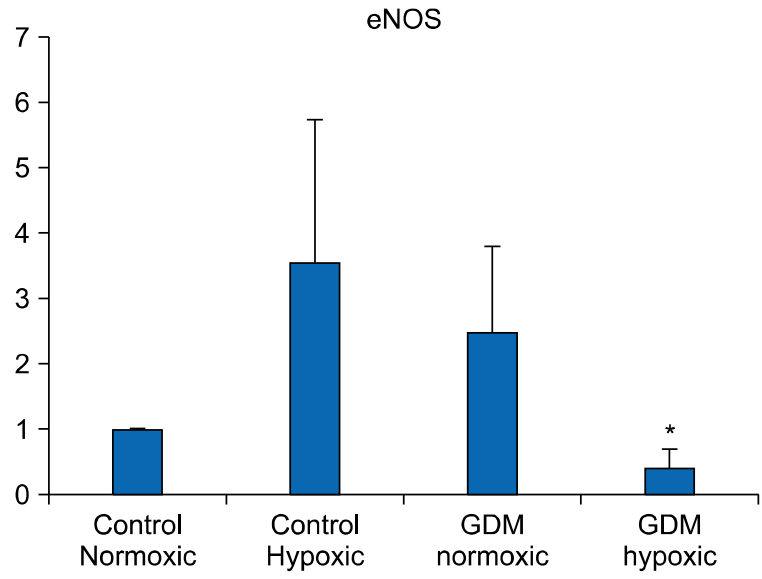

Fig. 1. Real-time PCR reactions were performed for endothelial specific markers ((A) VE-cadherin $[C D H 5$, vascular endothelial-cadherin, $\mathrm{CdH}$ 5, $\mathrm{Ca}^{++}$-dependent cell adhesion molecule, CD144], (B) endothelial nitric oxide synthase [eNOS] and (C) endothelial cell adhesion molecule [PECAM; CD 31]) using "custom designed" SYBR green mix as previously described. The data were analyzed in duplicated using the "modified" $2^{-\Delta \Delta C T}$ equation. $X$ axis is representing group of samples; Control Normoxic (C-HUCB ECFC-N, human umbilical cord blood ECFCs obtained from control pregnancies and plated under normoxic condition), Control Hypoxic (C-HUCB ECFC-H, human umbilical cord blood ECFCs obtained from control pregnancies and plated under hypoxic condition), GDM normoxic (GDM-HUCB ECFC-N, human umbilical cord blood ECFCs obtained from GDM subjects and plated under normoxic condition), GDM Hypoxic (GDM-HUCB ECFC-H, human umbilical cord blood ECFCs obtained from GDM subjects and plated under hypoxic condition). $\mathrm{Y}$ axis is representing the results of modified" $2^{-\triangle \Delta C T}$. (B) *eNOS mRNA expression in GDM-HUCB ECFC-H significantly decreased if compare with C-HUCB ECFC-N, C-HUCB ECFC-H and GDM-HUCB ECFC-N $(p<0.05)$. 
each experimental group (C-HUCB ECFC-N, C-HUCB ECFC-H, GDM-HUCB ECFC-N, GDM-HUCB ECFC-H) were expressed PECAM1 (CD31), VE-cadherin (CD144) and eNOS mRNAs which demonstrate their endothelial origin (Fig. 1). Hypoxia did not influence PECAM 1 and VE-cadherin mRNA expressions in control and GDM subjects however, eNOS mRNA expression in GDM-HUCB ECFC significantly decreased if compare with C-HUCB ECFC-N and GDM-HUCB ECFC-N samples (Fig. 1). VEGFA mRNA expression in C-HUCB ECFC-H was found significantly increased if compare with their nor- moxic controls. VEGFA mRNA expressions were also tented to increase in GDM normoxic as well as hypoxic samples if compared with normoxic controls. Hypoxia did not affect significantly IGF-1 mRNA expressions however, IGF-1 expressions tented to increase in GDM samples (Fig. 2). ADM mRNA expression also did not influenced with hypoxia and GDM significantly however, RAMP 2 expression significantly decreased if compare with their normoxic and hypoxic controls (Fig. 2).
A

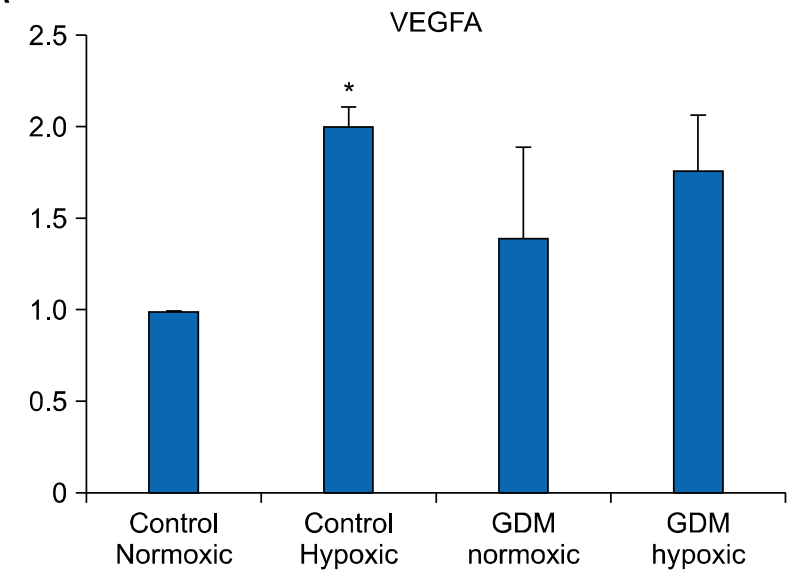

C

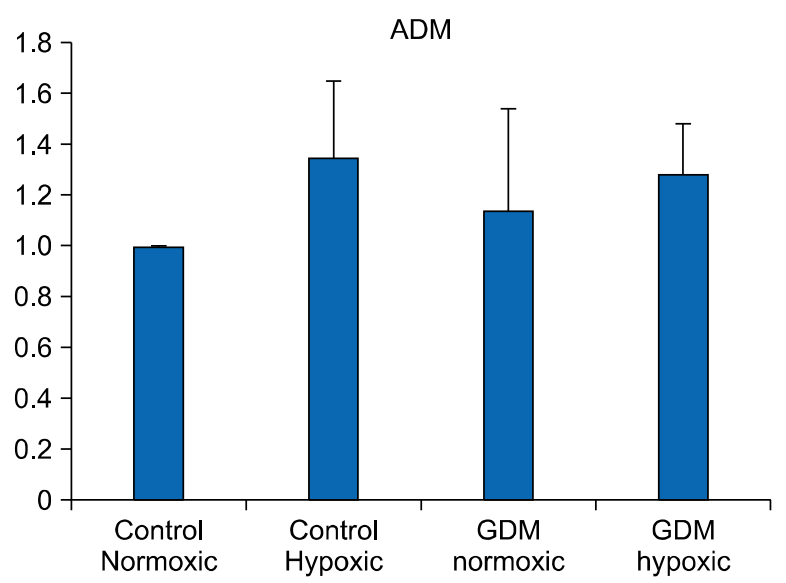

B

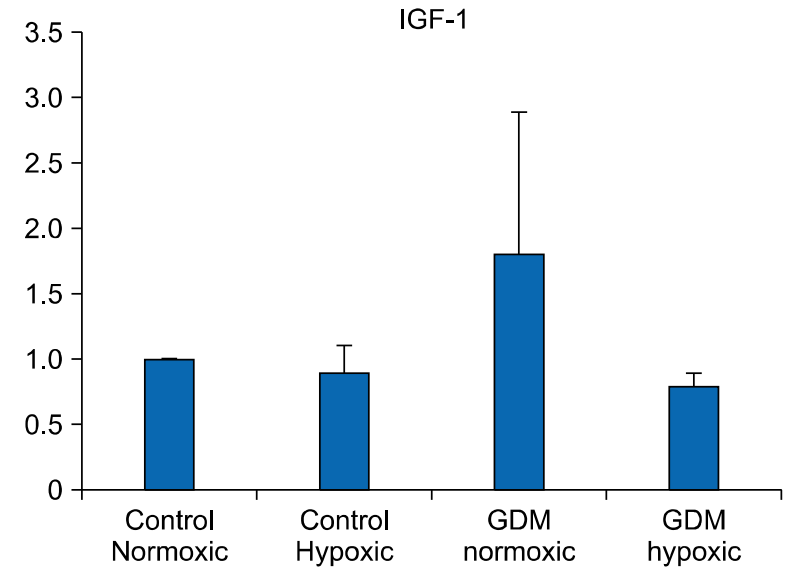

D

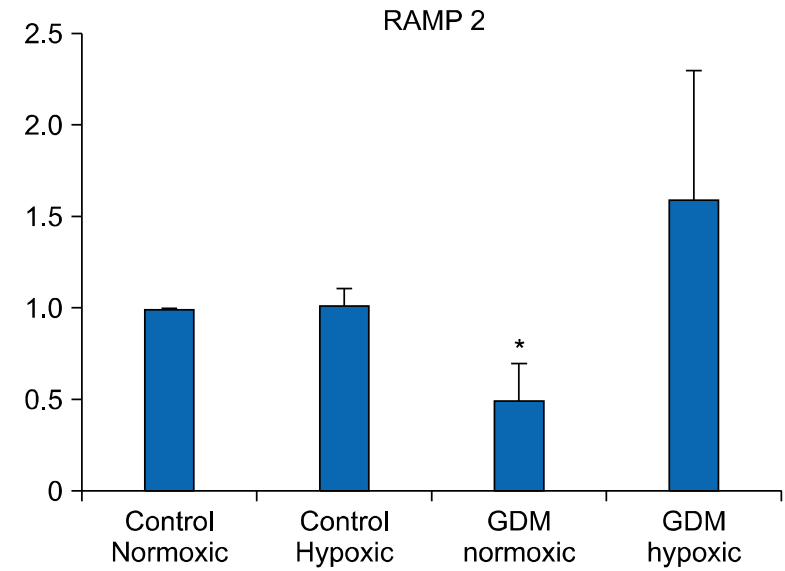

Fig. 2. Real-time PCR reactions were performed for pro-angiogenic markers ((A) vascular endothelial growth factor $A(V E G F A)$ and (B) insulin-like growth factor 1 [IGF-1]) and hypoxia specific markers ((C) adrenomedullin [ADM], (D) G protein coupled activity modifying protein 2 [RAMP 2]) using "custom designed" SYBR green mix as previously described. The data were analyzed in duplicated using the "modified" $2^{-\triangle \Delta C T}$ equation. $\mathrm{X}$ axis is representing group of samples: Control Normoxic (C-HUCB ECFC-N, human umbilical cord blood ECFCs obtained from control pregnancies and plated under normoxic condition), Control Hypoxic (C-HUCB ECFC-H, human umbilical cord blood ECFCs obtained from control pregnancies and plated under hypoxic condition), GDM normoxic (GDM-HUCB ECFC-N, human umbilical cord blood ECFCs obtained from GDM subjects and plated under normoxic condition), GDM Hypoxic (GDM-HUCB ECFC-H, human umbilical cord blood ECFCs obtained from GDM subjects and plated under hypoxic condition). Y axis is representing modified" $2^{-\triangle \triangle C T}$. (A) *VEGFA mRNA expression in control HUCB ECFCs which plated in vitro moderate hypoxic environment was found significantly increased if compare with their normoxic controls $(p<0.05)$. (D) *RAMP 2 mRNA expression was found significantly decreased if compare with their normoxic and hypoxic controls $(p<0.05)$. 
A Photomicrographs represents senescence assay

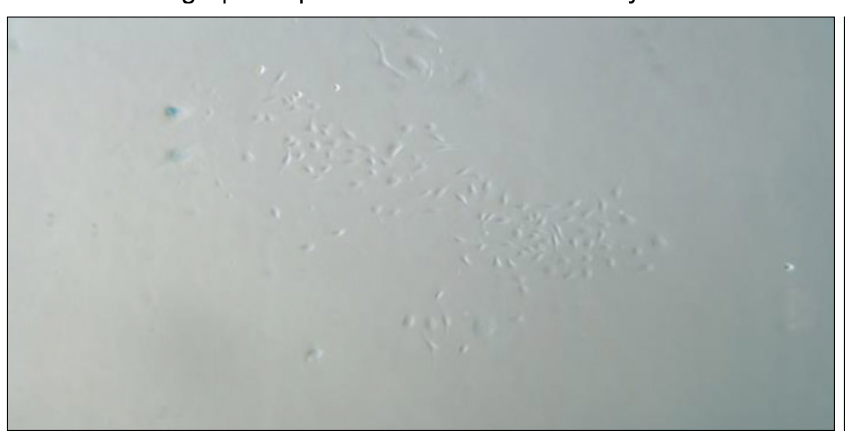

\section{C-HUCB ECFC-N}

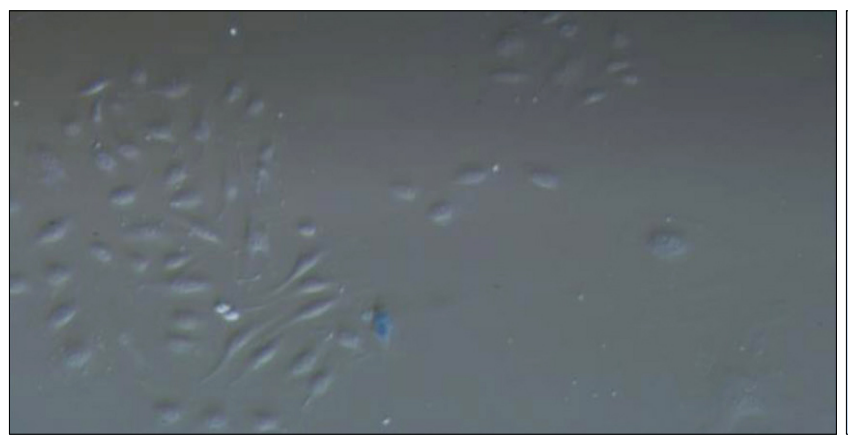

GDM-HUCB ECFC-N

B Quantitative assessment of senescence assay

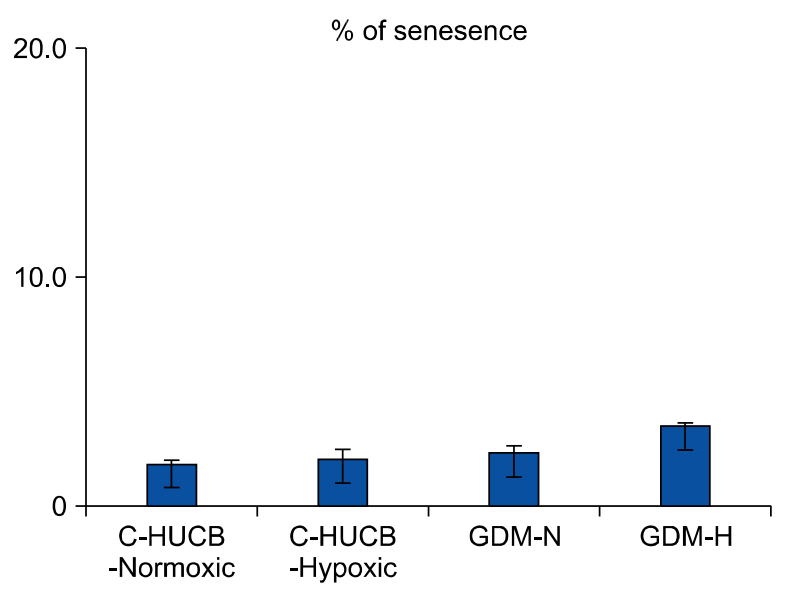

\section{Senescence Assay}

The photomicrographs in Fig. 3A represent senescence assay against $72 \mathrm{~h}$ hypoxia which indicates their clonogenic capacity. Fig. 3B represents their quantitative measurements of positively stained with SA $\beta$-gal of HUCB ECFCs. $72 \mathrm{~h}$ in vitro hypoxia $\left(5 \% \mathrm{CO}_{2} / 94 \% \mathrm{~N}_{2}\right.$ and $1 \%$ $\mathrm{O}_{2}$ in a triple gas incubator) did not cause any significant changes of clonogenic capacity of HUCB ECFCs obtained from control and GDM subjects.

\section{Matrigel Assay}

The photomicrographs represent matrigel assay, which

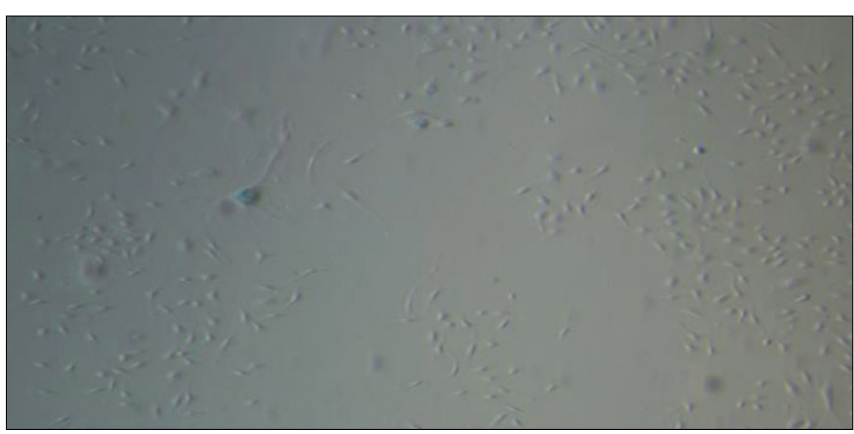

C-HUCB ECFC-H

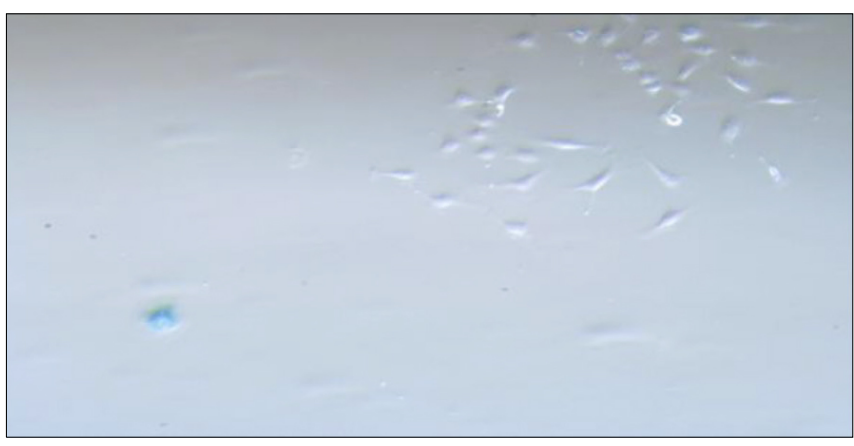

GDM-HUCB ECFC-H

Fig. 3. (A) Photomicrographs represents senescence assay against $72 \mathrm{~h}$ hypoxia; ECFCs derived from control ( $\mathrm{n}: 4)$ and GDM ( $\mathrm{n}$ : 4) subjects, which stained positively for SA $\beta$-Gal. (B) Quantitative assessment of senescence assay against $72 \mathrm{~h}$ hypoxia. ECFCs derived from control (n: 4) and GDM (n: 4) subjects, which stained positively for SA $\beta$-Gal and represents clonogenic capacity.

represents the vessel-forming ability (Fig. 4B). Their quantitative assessments demonstrated that a significant increase in capillary tube formation in control HUCB ECFCs with exposure to in vitro $72 \mathrm{~h}$ hypoxia (Fig. 4B). However, moderate hypoxia did not affect their capillary tube formation in GDM samples.

\section{Discussion}

Endothelium represents a defense barrier furthermore responds and integrates against pathological environment. The differentiation ability of endothelial progenitor cells 
A Photomicrographs represents matrigel assay
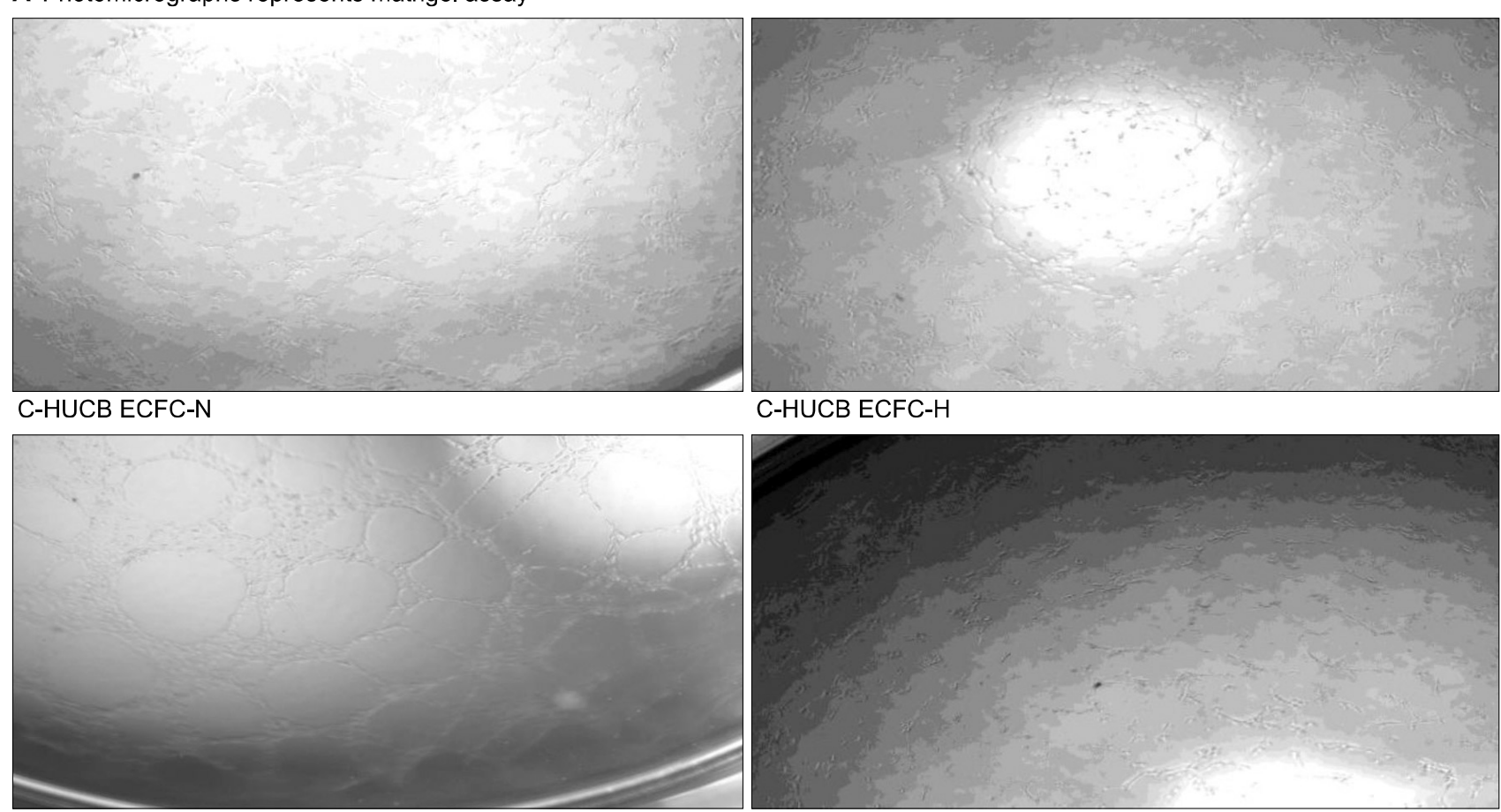

C-HUCB ECFC-H

\section{GDM-HUCB ECFC-N}

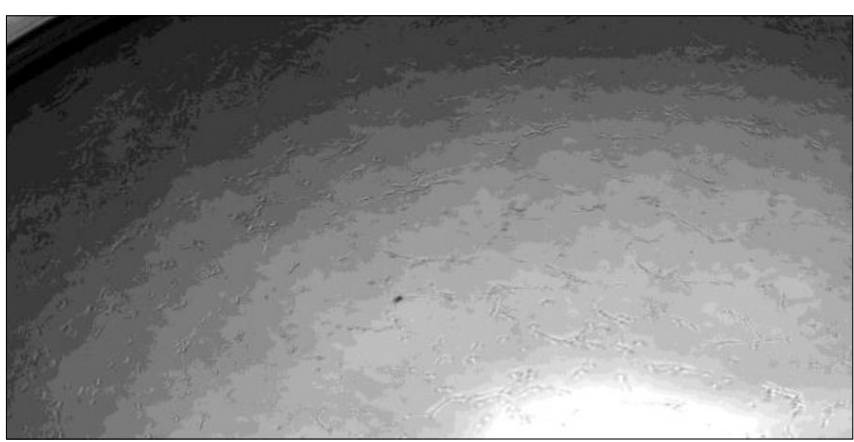

B Quantitative assessment of matrigel assay

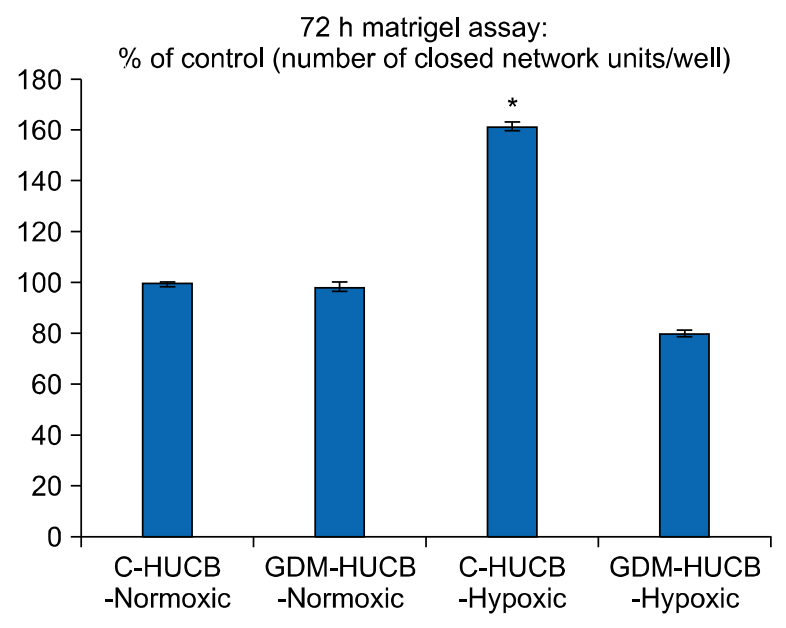

GDM-HUCB ECFC-H

Fig. 4. (A) Photomicrographs represents Matrigel Assay against 72 hours hypoxia. (B) Quantitative assessment of matrigel assay which represents tube formation. It was scoring the number of closed capillary tube networks per well. *Capillary tube formation in control HUCB ECFCs with exposure to in vitro $72 \mathrm{~h}$ hypoxia caused significantly increased if compared with C-HUCB ECFC-N, GDM-HUCB ECFC-N and GDM-HUCB ECFC-H ( $p$ $<0.01)$.

into the endothelial cells is clinically very important step to restore endothelial function. It also indicates healthy vascular flow. Indeed, understanding of human pluripotent stem cells (hPSCs) and their clonogenic and tissue regeneration capacity were generated brand new therapeutic approaches for cardiovascular diseases in recent years (1). Demonstration of larger amount of EPCs mobilization into the peripheral blood and their direct contribution to revascularization process was generated brand new ideas the treatment of many diseases such as stroke, cardiovascular disease, limb injury, ischemic ophthalmic

disease or hypoxic pulmonary artery diseases.

Previously we investigated that the adaptation ability and/or deficiency of EPCs against chronic hypoxia and fetal diabetic environment (11). They exhibited decreased proliferative capacity and diminished vessel forming ability. Chronic hypoxia caused impaired EPCs adaptation capability. Furthermore, GDM itself caused reduced proliferation capacity and vessel forming ability. It indicates that subjects from GDM mother highly possible increased cardiovascular risk during their adulthood or further decades (11). However, this project mainly de- 
signed to investigate endothelial progenitor cells adaptation ability against moderate hypoxic environment. Duration of hypoxia is one of the critical factor for development of vessel proliferation, migration and survival which called angiogenesis. Basically, the duration of hypoxia is the key moderator for angiogenic switch. Short duration of hypoxia initiates new vessel formation however long term hypoxia causes apoptosis. Consequently, the treatment modalities display opposite approaches in ischemic associated cardiovascular disease based on new vessel formation via their endothelial progenitors and tumor angiogenesis. Indeed, anti-angiogenic drug treatments have demonstrated that highly successful and beneficial results. We previously also demonstrated that fetal exposure to diabetic intrauterine environment affects EPCs adaptation ability. It indicates whether gestational diabetes generates higher cardiovascular risk for their adult offspring in their lifetime.

All samples in each experimental group were expressed endothelial specific markers which demonstrate their endothelial origin (Fig. 1). Hypoxia did not influence PECAM 1 and VE-cadherin mRNA expressions in control and GDM subjects however; eNOS mRNA expression was significantly decreased in hypoxic GDM samples (Fig. 1B). Endothelial cells release a short-lived vasodilator agent which called nitric oxide via eNOS and eNOS expression represents healthy endothelium because plays an important role reducing cardiovascular disease risk and development. However, some part related with vessel proliferation, migration and survival thus angiogenesis. Differentiation of endothelial progenitors to endothelial cells via generation of new intact endothelium requires functional endothelial progenitors which able to replace with injured vascular cells. Decreased eNOS mRNA expression in endothelial progenitors in GDM subjects indicates that whenever they replace with injured vascular endothelium they are not able to release nitric oxide via eNOS to display vasodilatation as normal healthy endothelial cells under ischemic conditions. It also indicates that the new vessel establishment from those cells which does not able to generate new vessel formation and development as healthy cells.

When we performed pro-angiogenic and hypoxia specific markers, moderate hypoxia caused increased in VEGFA expression in endothelial progenitors which obtained from normal subjects (Fig. 2A). VEGFA is important regulator for new vessel development and establishment, which plays respectable effort to angiogenic switch and initiates new vessel formation against ischemia. Endothelial cells able to adapt pathological environment and produce pro-an- giogenic and hypoxic regulators such as VEGFA, IGF-1, ADM, HIF-1 $\alpha$. Endothelial cells also produce different metabolites such as reactive oxygen species and lactate (22-24). Insufficient perfusion such as hypoxia, anemia or neoplasia trigger angiogenic switch to re-establish vessel flow. Increased VEGFA expression in endothelial progenitors which obtained from normal subjects indicates that whenever those cells differentiated to intact endothelial cells they are able to re-establish new vessel formation. However, if we compare endothelial progenitors which obtained with GDM subjects they are not able to generate new vessel formation to start collateral vessel development as with healthy endothelial progenitors when they differentiated to intact endothelial cells.

Hypoxia-inducible factor $1 \alpha$ (HIF $1 \alpha$ ) dependent pathway plays important role new vessel development against hypoxic environment such as tissue hypoxia. HIF 1 is a heterodimeric DNA binding complex, is a transcription factor, which composes of two basic helix-loop-helix domains; $\alpha$ ( $1 \alpha$ or $2 \alpha)$ domain which regulates by oxygen thus accepts as hypoxia inducible domain however, $1 \beta$ domain accepts as non-oxygen responsive subunit and expresses constitutively $(24,25)$. Furthermore, ADM and calcitonin receptor-like protein (CLRP) upregulate with hypoxia inducible HIF $1 \alpha$ and they bind to their receptors. We did not find any significant changes when we performed hypoxia specific markers in endothelial progenitors obtained from normal and GDM subjects under hypoxic conditions. The role of HIF-1 $\alpha$ transcription factor onto hypoxia associated angiogenesis and downstream pathway is important if ADM and CLRP pathways were triggered by hypoxia. Thus, we did not check HIF-1 $\alpha$ expression.

$\mathrm{ADM}$ binds to $\mathrm{ADM}$ receptors (ADMR1; CLR/RAMP2 and ADMR2; CLR/RAMP3) and named calcitonin receptor-like receptors (CLRs) $(24,25)$. They are member of the 7-transmembrane domain G-protein coupled receptor superfamily and require modulating proteins with a single transmembrane domain known as receptor activity modifying proteins (RAMP) $(24,25)$. Three RAMPs have been identified in the human genome and when they bind to CLR in the endoplasmic reticulum they actually transport to the plasma membrane. We designed several sets of gene specific primers for CLR and RAMP subtypes (1, 2 and 3). However, CLRP and RAMP 3 did not express in endothelial progenitor cells which obtained from human cord blood; CRLRP sense CCTGACATCACCAAGTGTGG and antisense AGTCAACTGCCCCAATCAAG (NM_005795) and RAMP3 sense CCCAAGCTCTTTGCTCATTC and antisense TAGTCCAAGCAGGGCCTAGA 
(accession number NM_005856). Possible reasons, CLRP normally presence in endoplasmic reticulum instead of the plasma membrane and isolation of their mRNAs could require different technics or endothelial progenitor cells which obtained from human cord blood do not express CLRP. The CLRP molecules transported by RAMP 1 to the membrane surface as a mature glycoprotein and represents as calcitonin gene related peptide receptor (CGRP) however, transported by RAMP 2 and RAMP 3 as a core-glycosylated adrenomedullin receptor (ADMR) which known as ADMR1 (CLR/RAMP2) and ADMR2 (CLR/RAMP3) respectively $(24,25)$. It is known that RAMP2 and RAMP3 are actually responsible for ADM's pharmacologic effects instead of CGRP (25). Although the more abundant expression isoform is RAMP2 however some factors could cause to elevate RAMP3 expression (25). We first of all found that RAMP2 was expressed all the samples and the second, RAMP 2 mRNA expression was significantly decreased in GDM endothelial progenitor cells if compare with their normoxic and hypoxic controls. Pregnancy, diabetes and associated complications cause to increase ADM levels $(28,29)$. However we did not find any significant differences ADM expressions in HUCB-ECFCs obtained from GDM subjects if compared with normal pregnancies under normoxic as well as hypoxic conditions (Fig. 2C). On the other hand, abundant form of RAMP 2 expression decreased in GDM subject. Underlined mechanisms further need to investigate.

Quantitative assessments of capillary tube formation demonstrated that moderate hypoxia significantly increased in vessel forming ability of endothelial progenitors obtained from normal pregnancies with unchanged their clonogenic capacity. If we combined with VEGFA expression, which significantly increased in endothelial progenitors obtained from normal subjects against hypoxia we could conclude that, VEGFA is also important regulator for new vessel development and angiogenic switch in endothelial progenitors as endothelium against ischemia. It indicates that whenever those endothelial progenitor cells differentiated to intact endothelial cells they are actually able to start new vessel development and angiogenic switch against ischemia as healthy endothelial cells. Thus, our result increased in VEGFA expression in HUCB ECFCs via hypoxia supports the information is during different pathological environment a larger amount of endothelial progenitors release and mobilize into the peripheral blood to generate a new vessel formation to re-establish blood flow.

HUCB ECFCs truly considers human pluripotent stem cells which display highly respectable vessel regeneration potential in ischemic vascular diseases. ECFCs obtained from human cord blood could replace with any injured vascular cells in ischemia and able to normalize blood flow. Initiation of re-vascularization and vascular repair require functional endothelial progenitors. For example, we did not find any differences vessel forming ability and VEGFA expression of HUCB-ECFC in GDM subjects under hypoxic environment. First of all, it demonstrates that their endothelial repair and development ability not good as endothelial progenitors obtained from normal subjects in hypoxic vascular diseases seen in cardiovascular disease, metabolic syndrome and diabetes. GDM has long term health consequences for her adult offspring inducing development of hypertension and premature cardiovascular disease $(11,30)$. Second, their self-repopulating capacity is not good as normal endothelial progenitors during hypoxic condition. It gives also useful information regarding GDM itself generates higher cardiovascular risk in their adult offspring via intrauterine affected EPCs with fetal exposure to gestational diabetes.

These results could generate different treatment modalities in cardiovascular complications, diabetes and metabolic syndrome. It also illuminates the role of endothelial progenitor cells onto future potential therapeutic strategies such as ischemia, coronary artery disease, pulmonary hypertension, obstructive hypoxic lung disease and sleep apnea disorders.

\section{Author Contribution}

U. Deniz Dincer MD., PhD., Professor, Chair designed the experiment, analyzed the data and wrote the manuscript. This work was completed in "The Herman B Wells Center", Indiana University School of Medicine, Department of Pediatrics, IU, Indianapolis, Indiana, USA (Dr. L. Haneline's laboratory).

\section{Potential conflict of interest}

There is no conflict of interest (any financial relationship and/or obligations) with any research sponsor, universities, scientific, government and other companies.

\section{References}

1. Prasain N, Lee MR, Vemula S, Meador JL, Yoshimoto M, Ferkowicz MJ, Fett A, Gupta M, Rapp BM, Saadatzadeh MR, Ginsberg M, Elemento O, Lee Y, Voytik-Harbin SL, Chung HM, Hong KS, Reid E, O'Neill CL, Medina RJ, Stitt AW, Murphy MP, Rafii S, Broxmeyer HE, Yoder MC. Differentiation of human pluripotent stem cells to cells similar to cord-blood endothelial colony-forming cells. Nat Biotechnol 2014;32:1151-1157 
2. Asahara T, Murohara T, Sullivan A, et al. Isolation of putative progenitor endothelial cells for angiogenesis. Science. 1997;275:964-967

3. Ghani U, Shuaib A, Salam A, Nasir A, Shuaib U, Jeerakathil T, Sher F, O'Rourke F, Nasser AM, Schwindt B, Todd K. Endothelial progenitor cells during cerebrovascular disease. Stroke 2005;36:151-153

4. Liew A, Barry F, O'Brien T. Endothelial progenitor cells: diagnostic and therapeutic considerations. Bioessays 2006; 28:261-270

5. Yoder MC, Mead LE, Prater D, Krier TR, Mroueh KN, Li F, Krasich R, Temm CJ, Prchal JT, Ingram DA. Redefining endothelial progenitor cells via clonal analysis and hematopoietic stem/progenitor cell principals. Blood 2007;109: 1801-1809

6. Kim J, Shapiro L, Flynn A. The clinical application of mesenchymal stem cells and cardiac stem cells as a therapy for cardiovascular disease. Pharmacol Ther. 2015;151:8-15

7. Loomans CJ, de Koning EJ, Staal FJ, Rookmaaker MB, Verseyden C, de Boer HC, Verhaar MC, Braam B, Rabelink TJ, van Zonneveld AJ. Endothelial progenitor cell dysfunction: a novel concept in the pathogenesis of vascular complications of type 1 diabetes. Diabetes 2004;53:195-199

8. Imanishi T, Hano T, Matsuo Y, Nishio I. Oxidized lowdensity lipoprotein inhibits vascular endothelial growth factor-induced endothelial progenitor cell differentiation. Clin Exp Pharmacol Physiol 2003;30:665-670

9. Imanishi T, Moriwaki C, Hano T, Nishio I. Endothelial progenitor cell senescence is accelerated in both experimental hypertensive rats and patients with essential hypertension. J Hypertens 2005;23:1831-1837

10. Hill JM, Zalos G, Halcox JP, Schenke WH, Waclawiw MA, Quyyumi AA, Finkel T. Circulating endothelial progenitor cells, vascular function, and cardiovascular risk. N Engl J Med 2003;348:593-600

11. Dincer UD. Fetal exposure to a diabetic intrauterine environment resulted in a failure of cord blood endothelial progenitor cell adaptation against chronic hypoxia. Stem Cells Cloning 2014;8:1-14

12. Blue EK, DiGiuseppe R, Derr-Yellin E, Acosta JC, Pay SL, Hanenberg H, Schellinger MM, Quinney SK, Mund JA, Case J, Haneline LS. Gestational diabetes induces alterations in the function of neonatal endothelial colony-forming cells. Pediatr Res 2014;75:266-272

13. Yoder MC. Cord blood banking and transplantation: advances and controversies. Curr Opin Pediatr 2014;26:163168

14. Timmermans F, Plum J, Yöder MC, Ingram DA, Vandekerckhove B, Case J. Endothelial progenitor cells: identity defined? J Cell Mol Med 2009;13:87-102

15. Prater DN, Case J, Ingram DA, Yoder MC. Working hypothesis to redefine endothelial progenitor cells. Leukemia 2007;21:1141-1149
16. Paternotte E, Gaucher C, Labrude P, Stoltz JF, Menu P. Review: behaviour of endothelial cells faced with hypoxia. Biomed Mater Eng 2008;18:295-299

17. Hwang HS, Maeng YS, Kim YH, Kwon YG, Park YW, Kim IK. Nestin expression during differentiation of fetal endothelial progenitor cells and hypoxic culture of human umbilical vein endothelial cells. Acta Obstet Gynecol Scand 2008;87:643-651

18. Ingram DA, Lien IZ, Mead LE, Estes $M$, Prater DN, Derr-Yellin E, DiMeglio LA, Haneline LS. In vitro hyperglycemia or a diabetic intrauterine environment reduces neonatal endothelial colony-forming cell numbers and function. Diabetes 2008;57:724-731

19. Gavard J. Endothelial permeability and VE-cadherin: a wacky comradeship. Cell Adh Migr 2013;7:455-461

20. Rijcken E, Mennigen RB, Schaefer SD, Laukoetter MG, Anthoni C, Spiegel HU, Bruewer M, Senninger N, Krieglstein CF. PECAM-1 (CD 31) mediates transendothelial leukocyte migration in experimental colitis. Am J Physiol Gastrointest Liver Physiol 2007;293:G446-G452

21. Jafarifar F, Yao P, Eswarappa SM, Fox PL. Repression of VEGFA by CA-rich element-binding microRNAs is modulated by hnRNP L. EMBO J 2011;30:1324-1334

22. Leung A, Ciau-Uitz A, Pinheiro P, Monteiro R, Zuo J, Vyas $\mathrm{P}$, Patient R, Porcher C. Uncoupling VEGFA functions in arteriogenesis and hematopoietic stem cell specification. Dev Cell 2013;24:144-158

23. Gombos A, Metzger-Filho O, Dal Lago L, Awada-Hussein A. Clinical development of insulin-like growth factor receptor--1 (IGF-1R) inhibitors: at the crossroad? Invest New Drugs 2012;30:2433-2442

24. Wong HK, Tang F, Cheung TT, Cheung BM. Adrenomedullin and diabetes. World J Diabetes 2014;5:364-371

25. Larráyoz IM, Martínez-Herrero S, García-Sanmartín J, Ochoa-Callejero L, Martínez A. Adrenomedullin and tumour microenvironment. J Transl Med 2014;12:339

26. Dincer UD, Araiza AG, Knudson JD, Molina PE, Tune JD. Sensitization of coronary alpha-adrenoceptor vasoconstriction in the prediabetic metabolic syndrome. Microcirculation 2006;13:587-595

27. Dincer UD, Araiza A, Knudson JD, Shao CH, Bidasee KR, Tune JD. Dysfunction of cardiac ryanodine receptors in the metabolic syndrome. J Mol Cell Cardiol 2006;41:108-114

28. Dincer UD. Cardiac $\beta$-adrenoceptor expression is markedly depressed in Ossabaw swine model of cardiometabolic risk. Int J Gen Med 2011;4:493-499

29. Livak KJ, Schmittgen TD. Analysis of relative gene expression data using real-time quantitative PCR and the 2(-Delta Delta C(T)) Method. Methods 2001;25:402-408

30. Dincer UD. Human endothelial progenitor cell application in vascular diseases seen in metabolic syndrome. J Metabolic Synd 2015;4:e115 